\title{
Note on some greatest common divisor matrices
}

\author{
by
}

\author{
Peter Lindqvist and Kristian Seip (Trondheim)
}

Some quadratic forms related to "greatest common divisor matrices" are represented in terms of $L^{2}$-norms of rather simple functions. Our formula is especially useful when the size of the matrix grows, and we will study the asymptotic behaviour of the smallest and largest eigenvalues. Indeed, a sharp bound in terms of the zeta function is obtained. Our leading example is a hybrid between Hilbert's matrix and Smith's matrix.

1. Introduction. While studying a question about Riesz bases we found an unexpected analytic connection to certain matrices or quadratic forms. A specific example is

$$
\left(\begin{array}{llllll}
\frac{1}{1 \cdot 1} & \frac{1}{1 \cdot 2} & \frac{1}{1 \cdot 3} & \frac{1}{1 \cdot 4} & \frac{1}{1 \cdot 5} & \frac{1}{1 \cdot 6} \\
\frac{1}{2 \cdot 1} & \frac{4}{2 \cdot 2} & \frac{1}{2 \cdot 3} & \frac{4}{2 \cdot 4} & \frac{1}{2 \cdot 5} & \frac{4}{2 \cdot 6} \\
\frac{1}{3 \cdot 1} & \frac{1}{3 \cdot 2} & \frac{9}{3 \cdot 3} & \frac{1}{3 \cdot 4} & \frac{1}{3 \cdot 5} & \frac{9}{3 \cdot 6} \\
\frac{1}{4 \cdot 1} & \frac{4}{4 \cdot 2} & \frac{1}{4 \cdot 3} & \frac{16}{4 \cdot 4} & \frac{1}{4 \cdot 5} & \frac{4}{4 \cdot 6} \\
\frac{1}{5 \cdot 1} & \frac{1}{5 \cdot 2} & \frac{1}{5 \cdot 3} & \frac{1}{5 \cdot 4} & \frac{25}{5 \cdot 5} & \frac{1}{5 \cdot 6} \\
\frac{1}{6 \cdot 1} & \frac{4}{6 \cdot 2} & \frac{9}{6 \cdot 3} & \frac{4}{6 \cdot 4} & \frac{1}{6 \cdot 5} & \frac{36}{6 \cdot 6}
\end{array}\right)
$$

The numerators are the squares of the greatest common divisors of the row and column numbers. Our observation is that the corresponding quadratic form is equal to a norm, viz.

$$
\begin{aligned}
\int_{0}^{\pi}\left|\sum_{k=1}^{\infty} \frac{c_{1} \sin k x+c_{2} \sin 2 k x+\ldots+c_{6} \sin 6 k x}{k}\right|^{2} d x & \\
= & \frac{\pi^{3}}{12} \sum_{m, n=1}^{6} \frac{(\operatorname{gcd}(m, n))^{2}}{m n} c_{m} \bar{c}_{n} .
\end{aligned}
$$

1991 Mathematics Subject Classification: 11C20, 11M41, 11A05. 
Since

$$
\frac{\pi-x}{2}=\sum_{k=1}^{\infty} \frac{\sin k x}{k} \quad(0<x<2 \pi)
$$

a rather elementary characterization is possible.

Returning to the general case, we find a historical remark appropriate. The determinant $\operatorname{det}(\operatorname{gcd}(m, n))$ of size $N \times N$ was evaluated by H. Smith in 1876. The $m, n$ entry of the determinant is the greatest common divisor of $m$ and $n$. According to $[\mathrm{S}]$, the value of the determinant is $\varphi(1) \varphi(2) \ldots \varphi(N)$, where $\varphi$ is Euler's totient function. Smith also gave formulas for more general determinants like $\operatorname{det}\left(\operatorname{gcd}^{s}(m, n)\right)$, where $s$ is any exponent. See also pp. 264-267 in the third edition of the book [NZ]. The corresponding matrix has been studied; see [BL], [C] and the references given there.

The matrix $(\operatorname{gcd}(m, n))_{N \times N}$ has been explicitly written as the product of two triangular matrices and the inverse matrix has been constructed (cf. $[\mathrm{S}]$ and $[\mathrm{BL}])$. These results are readily extended to our matrices, but that is not our concern now. However, the eigenvalues do not seem to be known. We will use our recent work [HLS] about Riesz bases to study their asymptotic behaviour as $N \rightarrow \infty$. Somewhat surprisingly, a sharp bound is given in the Theorem in Section 3. Such estimates are not easy to come by, in general.

2. The functions $u(x), u(2 x), u(3 x), \ldots$ Consider the function

$$
u(x)=\sum_{k=1}^{\infty} \frac{\sin k x}{k^{s}},
$$

where $s>1 / 2$. (One can also allow $s$ to be a complex number.) In passing, let us mention that for $s=1,3,5, \ldots$ this is essentially just a well-known Bernoulli polynomial. Define the functions $u_{1}(x)=u(x), u_{2}(x)=u(2 x)$, $u_{3}(x)=u(3 x), \ldots$ A finite number of these functions are linearly independent. Consider the usual inner product in $L^{2}(0, \pi)$. We have

$$
\begin{aligned}
\left\langle u_{m}, u_{n}\right\rangle & =\int_{0}^{\pi} u_{m}(x) u_{n}(x) d x=\sum_{k=1}^{\infty} \sum_{l=1}^{\infty} k^{-s} l^{-s} \int_{0}^{\pi} \sin (k m x) \sin (\ln x) d x \\
& =\frac{\pi}{2} \sum_{\substack{k, l=1 \\
k m=l n}}^{\infty} \frac{1}{k^{s} l^{s}} .
\end{aligned}
$$

In order to evaluate the last sum, let $d=\operatorname{gcd}(m, n)$. The possible values of the summation indices are

$$
k=\frac{n \nu}{d}, \quad l=\frac{m \nu}{d} \quad(\nu=1,2,3, \ldots)
$$

so that $k m=l n$. 
This yields

$$
\left\langle u_{m}, u_{n}\right\rangle=\frac{\pi}{2} \zeta(2 s) \frac{(\operatorname{gcd}(m, n))^{2 s}}{m^{s} n^{s}},
$$

where $\zeta(2 s)=1+2^{-2 s}+3^{-2 s}+\ldots$ is Riemann's zeta function.

The usual $L^{2}(0, \pi)$-norm of the linear combination $c_{1} u_{1}(x)+\ldots+c_{N} u_{N}(x)$ is easy to calculate. Indeed,

$$
\begin{aligned}
\left\|c_{1} u_{1}+\ldots+c_{N} u_{N}\right\|_{2}^{2} & =\sum_{m, n=1}^{N} c_{m} \bar{c}_{n}\left\langle u_{m}, u_{n}\right\rangle \\
& =\frac{\pi}{2} \zeta(2 s) \sum_{m, n=1}^{N} \frac{(\operatorname{gcd}(m, n))^{2 s}}{m^{s} n^{s}} c_{m} \bar{c}_{n}
\end{aligned}
$$

when $s>1 / 2$. The subsequent results are based on this fundamental observation.

From this representation one can read off that the matrix

$$
M_{s}=\left(\frac{(\operatorname{gcd}(m, n))^{2 s}}{m^{s} n^{s}}\right)_{N \times N}
$$

is positive definite when $s>1 / 2$. The same concerns the matrix

$$
S_{s}=\left((\operatorname{gcd}(m, n))^{2 s}\right)_{N \times N},
$$

when $s>1 / 2$. (To see this, replace $c_{n}$ by $n^{s} c_{n}$ in the quadratic form.) The usual proof of this fact is combinatorial. From an arithmetic point of view, these matrices are of the same kind, also when $s \leq 1 / 2$. Unfortunately, our characterization above of the quadratic form as a norm breaks down when $s \leq 1 / 2$. This is due to the divergence of the series defining $u=u(x)$.

Choosing $c_{m}$ equal to zero when $m$ is not a prime number, we have an interesting submatrix. Some other interesting quadratic forms involving greatest common divisors can be given a similar norm characterization. Starting with the function

$$
u(x)=\sum_{k=1}^{\infty} a_{k} \sin k x
$$

and defining $u_{n}=u(n x), n=1,2,3, \ldots$, we arrive at

$$
\left\langle u_{m}, u_{n}\right\rangle=\frac{\pi}{2} \sum_{\substack{k, l=1 \\ k m=l n}}^{\infty} a_{k} \bar{a}_{l},
$$

at least formally. For example, fix $q$ so that $|q|<1$ and set $a_{k}=q^{k}$. Then we have a geometric series: 


$$
\begin{aligned}
\left\langle u_{m}, u_{n}\right\rangle & =\frac{\pi}{2} \sum_{\nu=1}^{\infty} q^{n \nu / d} q^{m \nu / d}=\frac{\pi}{2} q^{(n+m) / d}\left(1+q^{(n+m) / d}+q^{2(n+m) / d}+\ldots\right) \\
& =\frac{\pi}{2} \cdot \frac{q^{(n+m) / d}}{1-q^{(n+m) / d}},
\end{aligned}
$$

where $d=\operatorname{gcd}(m, n)$. In conclusion,

$$
\left\|c_{1} u_{1}+\ldots+c_{N} u_{N}\right\|_{2}^{2}=\frac{\pi}{2} \sum_{m, n=1}^{N} \frac{q^{(n+m) / \operatorname{gcd}(n, m)}}{1-q^{(n+m) / \operatorname{gcd}(n, m)}} c_{n} \bar{c}_{m} .
$$

In particular, the choice $q=1 / 2$ gives us the positive definite matrix

$$
Q=\left(\frac{1}{2^{(n+m) / \operatorname{gcd}(n, m)}-1}\right)_{N \times N} .
$$

Needless to say, many other matrices, occasionally of interest in Number Theory, can be obtained in this way. Instead of the trigonometric system one can use other systems, orthogonal with a weight function.

3. Asymptotic bounds for the eigenvalues. We now turn to the asymptotic behaviour of the eigenvalues of the matrix

$$
M_{s}=\left(\frac{(\operatorname{gcd}(m, n))^{2 s}}{m^{s} n^{s}}\right)_{N \times N}
$$

encountered above. This seems to be a delicate question and we shall rely upon the theory of analytic functions. Let $\lambda_{N}(s)$ denote the smallest eigenvalue and $\Lambda_{N}(s)$ the largest eigenvalue of the matrix $M_{s}$ of size $N \times N$.

THEOREM. We have

$$
\frac{\zeta(2 s)}{\zeta(s)^{2}} \leq \lambda_{N}(s) \leq \Lambda_{N}(s) \leq \frac{\zeta(s)^{2}}{\zeta(2 s)},
$$

when $s>1$. The bounds are sharp in the sense that

$$
\liminf _{N \rightarrow \infty} \lambda_{N}(s)=\frac{\zeta(2 s)}{\zeta(s)^{2}}, \quad \limsup _{N \rightarrow \infty} \Lambda_{N}(s)=\frac{\zeta(s)^{2}}{\zeta(2 s)},
$$

when $s>1$. In the case $1 / 2<s \leq 1$ we have

$$
\liminf _{N \rightarrow \infty} \lambda_{N}(s)=0, \quad \limsup _{N \rightarrow \infty} \Lambda_{N}(s)=\infty .
$$

Proof. The inequalities are equivalent to

$$
\zeta(2 s) \zeta(s)^{-2} \sum_{n=1}^{N}\left|c_{n}\right|^{2} \leq \sum_{m, n=1}^{N} \frac{(\operatorname{gcd}(m, n))^{2 s}}{m^{s} n^{s}} c_{m} \bar{c}_{n} \leq \zeta(s)^{2} \zeta(2 s)^{-1} \sum_{n=1}^{N}\left|c_{n}\right|^{2}
$$


when $s>1$. Recall that the functions $u_{1}, u_{2}, u_{3}, \ldots$ form a Riesz basis in $L^{2}(0, \pi)$, by definition, if and only if

$$
\alpha^{2} \sum_{n=1}^{N}\left|c_{n}\right|^{2} \leq\left\|c_{1} u_{1}+\ldots+c_{N} u_{N}\right\|_{2}^{2} \leq \beta^{2} \sum_{n=1}^{N}\left|c_{n}\right|^{2}
$$

when $N=1,2,3, \ldots, 0<\alpha^{2} \leq \beta^{2}<\infty$. According to our recent work about Riesz bases this holds when $s>1$, but not when $1 / 2<s \leq 1$ (cf. [HLS, Theorem 5.2]). Moreover, it follows from [HLS, Theorem 3.1] and the proof of [HLS, Theorem 5.2] that the constants

$$
\begin{aligned}
& \beta^{2}=\frac{\pi}{2} \sup _{\operatorname{Re} z>0}\left|\sum_{n=1}^{\infty} n^{-s-z}\right|^{2}=\frac{\pi}{2} \zeta(s)^{2}, \\
& \frac{1}{\alpha^{2}}=\frac{2}{\pi} \sup _{\operatorname{Re} z>0}\left|\sum_{n=1}^{\infty} n^{-s-z}\right|^{-2}=\frac{2 \zeta(s)^{2}}{\pi \zeta(2 s)^{2}}
\end{aligned}
$$

are sharp. The evaluation of $\alpha$ and $\beta$ comes from the Euler product

$$
\zeta(s+z)=\sum_{n=1}^{\infty} n^{-s-z}=\prod_{p}\left(1-p^{-s-z}\right)^{-1},
$$

where the factors behave as if they were independent, by Kronecker's approximation theorem. (See also [HLS, Corollary 5.3].) Note that

$$
\alpha^{2}=\frac{\pi}{2} \prod_{p}\left(1+p^{-s}\right)^{-2}=\frac{\pi}{2} \cdot \frac{\prod_{p}\left(1-p^{-s}\right)^{2}}{\prod_{p}\left(1-p^{-2 s}\right)^{2}} .
$$

The desired result follows from the characterization of the norm as a quadratic form in Section 2. This concludes our proof.

The above proof uses rather deep properties of Dirichlet series of complex variables. Because the sharp asymptotic bounds in the Theorem are not attained for any finite $N$, it does not seem to be likely that one could construct an "arithmetic proof".

Added in proof (February 1998). It has come to our attention that the fact that $\lim \sup \Lambda_{N}<\infty$ if and only if $s>1$ has been established on page 578 of A. Wintner, Diophantine approximations and Hilbert's space, Amer. J. Math. 66 (1944). Wintner did not give any sharp upper bound. We thank M. Balazard for drawing our attention to this reference.

\section{References}

[BL] K. Bourque and S. Ligh, On GCD and LCM matrices, Linear Algebra Appl. 174 (1992), 65-74.

[C] S. Z. Chun, GCD and LCM power matrices, Fibonacci Quart. 34 (1996), 290-297. 
[HLS] H. Hedenmalm, P. Lindqvist and K. Seip, A Hilbert space of Dirichlet series and systems of dilated functions in $L^{2}(0,1)$, Duke Math. J. 86 (1997), 1-37.

[NZ] I. Niven and H. Zuckerman, An Introduction to the Theory of Numbers, 3rd ed., Wiley, New York, 1960.

[S] H. Smith, On the value of a certain arithmetical determinant, Proc. London Math. Soc. 7 (1875-6), 208-212.

Department of Mathematics

Norwegian Institute of Technology

N-7034 Trondheim, Norway

E-mail: lqvist@math.ntnu.no

seip@math.ntnu.no 